Commun. Korean Math. Soc. 26 (2011), No. 4, pp. 557-573

http://dx.doi.org/10.4134/CKMS.2011.26.4.557

\title{
QUASI-ARMENDARIZ PROPERTY FOR SKEW POLYNOMIAL RINGS
}

\author{
Muhittin Başer and Tai Keun KwaK
}

\begin{abstract}
The concept of the quasi-Armendariz property of rings properly contains Armendariz rings and semiprime rings. In this paper, we extend the quasi-Armendariz property for a polynomial ring to the skew polynomial ring, hence we call such ring a $\sigma$-quasi-Armendariz ring for a ring endomorphism $\sigma$, and investigate its structures, several extensions and related properties. In particular, we study the semiprimeness and the quasi-Armendariz property between a ring $R$ and the skew polynomial ring $R[x ; \sigma]$ of $R$, and so these provide us with an opportunity to study quasi-Armendariz rings and semiprime rings in a general setting, and several known results follow as consequences of our results.
\end{abstract}

\section{Introduction}

Rege et al. called a ring $R$ Armendariz [18] if whenever the product of any two polynomials in $R[x]$ over $R$ is zero, then so is the product of any pair of coefficients from the two polynomials. This nomenclature was used by them since it was Armendariz [1, Lemma 1] who initially showed that a reduced ring always satisfies this condition. Such rings have been extensively studied in literature $[12,15,18]$. Armendariz rings are generalized to quasiArmendariz rings. A ring $R$ is called quasi-Armendariz [5] if whenever polynomials $f(x)=a_{0}+a_{1} x+\cdots+a_{m} x^{m}, g(x)=b_{0}+b_{1} x+\cdots+b_{n} x^{n} \in R[x]$ satisfy $f(x) R[x] g(x)=0$, then $a_{i} R b_{j}=0$ for each $i, j$. Semiprime rings are quasi-Armendariz rings by [5, Corollary 3.8], but the converse does not hold in general. In [5], it is shown that the class of quasi-Armendariz rings is Morita stable and that several extensions of a quasi-Armendariz ring are also quasiArmendariz rings. According to [7] and [10], the Armendariz property for a polynomial ring is extended to one for the skew polynomial ring which is a generalization of a $\sigma$-rigid ring. An endomorphism $\sigma$ of a ring $R$ is called rigid [13] if $a \sigma(a)=0$ implies $a=0$ for $a \in R$, and $R$ is called a $\sigma$-rigid ring [6] if there exists a rigid endomorphism $\sigma$ of $R$. Any rigid endomorphism of a ring

Received July 19, 2010

2010 Mathematics Subject Classification. 16S36, 16N60, 16W20.

Key words and phrases. quasi-Armendariz property, skew polynomial ring, semiprime ring, rigid ring. 
is a monomorphism, and $\sigma$-rigid rings are reduced rings (i.e., rings have not nonzero nilpotent elements) by [6, Proposition 5]. For an endomorphism $\sigma$ of a ring $R$, the skew polynomial ring $R[x ; \sigma]$ of $R$ consists of the polynomial in $x$ with coefficients in $R$ written on the left, subject to the relation $x r=\sigma(r) x$ for all $r \in R$. A ring $R$ is called $\sigma$-Armendariz (resp., $\sigma$-skew Armendariz) [10, Definition 1.1] (resp., [7, Definition]) if for $p(x)=\sum_{i=0}^{m} a_{i} x^{i}$ and $q(x)=\sum_{j=0}^{n} b_{j} x^{j}$ in $R[x ; \sigma], p(x) q(x)=0$ implies $a_{i} b_{j}=0$ (resp., $a_{i} \sigma^{i}\left(b_{j}\right)=0$ ) for all $0 \leq i \leq m$ and $0 \leq j \leq n$. Any $\sigma$-rigid ring is $\sigma$-Armendariz by [6, Proposition 6] and any $\sigma$-Armendariz ring is $\sigma$-skew Armendariz by [10, Theorem 1.8], but the converses do not hold by [10, Example 1.6 and Example 1.9]. Moreover, by [7, Proposition 3], [10, Proposition 1.7] and [16, Theorem A], $R$ is a $\sigma$-rigid ring if and only if $R$ is a reduced and $\sigma$-Armendariz ring if and only if $R$ is a reduced and $\sigma$-skew Armendariz ring for a monomorphism $\sigma$ if and only if the skew polynomial ring $R[x ; \sigma]$ of $R$ is a reduced ring. Various extensions of the extended Armendariz rings are also investigated in [7] and [10].

On the other hand, the notion of $\sigma$-skew Armendariz rings is generalized as follows: Let $\sigma$ be an endomorphism of a $\operatorname{ring} R$. $R$ is called a $\sigma$-skew quasi-Armendariz ring [9, Definition 2.1] if for $p(x)=\sum_{i=0}^{m} a_{i} x^{i}$ and $q(x)=$ $\sum_{j=0}^{n} b_{j} x^{j}$ in $R[x ; \sigma], p(x) R[x ; \sigma] q(x)=0$ implies $a_{i} R \sigma^{i}\left(b_{j}\right)=0$ for all $0 \leq$ $i \leq m$ and $0 \leq j \leq n$; while Cortes [4, Definition 3.11] used the term quasiskew Armendariz for what is called $\sigma$-skew quasi-Armendariz when $\sigma$ is an automorphism. It is shown that the class of $\sigma$-skew quasi-Armendariz rings is Morita stable and that several extensions of a $\sigma$-skew quasi-Armendariz ring are also $\sigma$-skew quasi-Armendariz rings in [4] and [9]. Observe that every $\sigma$ skew Armendariz ring is $\sigma$-skew quasi-Armendariz when $\sigma$ is an epimorphism, but the converse does not hold by [9, Example 2.2(1)].

In this paper, we introduce the concept of a $\sigma$-quasi-Armendariz ring (Definition 2.4), drawing a parallel with a $\sigma$-skew quasi-Armendariz ring. We show that for any endomorphism $\sigma$, every $\sigma$-Armendariz ring is $\sigma$-quasi-Armendariz, and every $\sigma$-quasi-Armendariz ring is $\sigma$-skew quasi-Armendariz in case that $\sigma$ is an epimorphism; but the converses do not hold. We also study the related topics and extensions of $\sigma$-quasi-Armendariz rings. In particular, we investigate the semiprimeness and the quasi-Armendariz property between $R$ and $R[x ; \sigma]$, and so these provide us with an opportunity to study quasi-Armendariz rings and semiprime rings in a general setting.

Throughout this paper $R$ denotes an associative ring with identity and $\sigma$ denotes a nonzero and non identity endomorphism, unless specified otherwise. Denote the $n$ by $n$ full matrix ring over $R$ by $\operatorname{Mat}_{n}(R)$ and the $n$ by $n$ upper triangular matrix ring over $R$ by $U_{n}(R)$. Let $\mathbb{Z}, \mathbb{Z}_{n}$ and $\mathbb{Q}$ be the set of all integers, the ring of integers modulo $n$ and the set of all rational numbers, respectively. 


\section{Structures of $\sigma$-quasi-Armendariz rings}

Note that for $p(x), q(x) \in R[x ; \sigma], p(x) R[x ; \sigma] q(x)=0$ if and only if $p(x) r x^{t} q(x)=0$ for any $r \in R$ and nonnegative integer $t$. We freely use this fact in the process. Consider the following condition $(*)$ :

(*) For $p(x)=\sum_{i=0}^{m} a_{i} x^{i}$ and $q(x)=\sum_{j=0}^{n} b_{j} x^{j}$ in $R[x ; \sigma], p(x) R[x ; \sigma] q(x)=$ 0 implies $a_{i} R[x ; \sigma] b_{j}=0$ for all $0 \leq i \leq m$ and $0 \leq j \leq n$, equivalently, $a_{i} R \sigma^{t}\left(b_{j}\right)=0$ for any nonnegative integer $t$ and all $i, j$.

Every $\sigma$-Armendariz ring satisfies the condition $(*)$ by simple computation, but the converse does not hold by Example 2.7 to follow, and there exists a $\sigma$-skew Armendariz ring $R$ which does not satisfy the condition $(*)$ by the next example.

Example 2.1. Let $R$ be the polynomial ring $\mathbb{Z}_{2}[x]$ over $\mathbb{Z}_{2}$, and let the endomorphism $\sigma: R \rightarrow R$ be defined by $\sigma(f(x))=f(0)$ for $f(x) \in \mathbb{Z}_{2}[x]$. Then $R$ is not $\sigma$-Armendariz, but $R$ is a reduced $\sigma$-skew Armendariz ring by [10, Example 1.9]. For $p(y)=x y=q(y)$ in $\mathbb{Z}_{2}[x][y ; \sigma]$, we have $p(y)\left(\mathbb{Z}_{2}[x][y ; \sigma]\right) q(y)=0$ but $0 \neq x f(x) x \in x\left(\mathbb{Z}_{2}[x][y ; \sigma]\right) x$ for any nonzero $f(x) \in \mathbb{Z}_{2}[x]$, showing that $R$ does not satisfy the condition $(*)$.

Recall that for an automorphism $\sigma$ of a ring $R, R$ is called quasi $\sigma$-rigid [8, Definition 1.3] if $a R \sigma(a)=0$ implies $a=0$ for $a \in R$. In [8], it is shown that every $\sigma$-rigid ring is quasi $\sigma$-rigid and every quasi $\sigma$-rigid ring is semiprime but not conversely.

Proposition 2.2. Let $\sigma$ be an automorphism of a ring $R$. If $R$ is a quasi $\sigma$-rigid ring, then $R$ satisfies the condition ( $*$ ).

Proof. Assume that $R$ is a quasi $\sigma$-rigid ring. Let $p(x)\left(r x^{t}\right) q(x)=0$ for any $r \in R$ and nonnegative integer $t$, where $p(x)=\sum_{i=0}^{m} a_{i} x^{i}$ and $q(x)=\sum_{j=0}^{n} b_{j} x^{j}$ in $R[x ; \sigma]$. We claim that $a_{i} R \sigma^{t}\left(b_{j}\right)=0$ for any nonnegative integer $t$ and all $0 \leq i \leq m$ and $0 \leq j \leq n$. We proceed by induction on $i+j$. From $p(x)\left(r x^{t}\right) q(x)=0$ for any $r \in R$ and nonnegative integer $t$, we get $a_{0} R \sigma^{t}\left(b_{0}\right)=$ 0 and so it proves for $i+j=0$. Now assume that our claim is true for $i+j \leq k-1$. For $i+j=k$, we have

$$
a_{0} r \sigma^{t}\left(b_{k}\right)+a_{1} \sigma(r) \sigma^{t+1}\left(b_{k-1}\right)+\cdots+a_{k} \sigma^{k}(r) \sigma^{t+k}\left(b_{0}\right)=0 .
$$

Multiplying Eq.(1) by $\sigma^{t+k}\left(b_{0}\right) R$ on the left hand-side, we have

$$
\begin{aligned}
\sigma^{t+k}\left(b_{0}\right) R a_{k} \sigma^{k}(r) \sigma^{t+k}\left(b_{0}\right)=0 & \Rightarrow\left(a_{k} R \sigma^{t+k}\left(b_{0}\right) R\right)^{2}=0 \\
& \Rightarrow a_{k} R \sigma^{t+k}\left(b_{0}\right)=0 \\
& \Rightarrow a_{k} R \sigma^{t}\left(b_{0}\right)=0
\end{aligned}
$$

for any nonnegative integer $t$, by the induction hypothesis and [8, Lemma 2.4]. Then Eq.(1) becomes

(2) $\quad a_{0} r \sigma^{t}\left(b_{k}\right)+a_{1} \sigma(r) \sigma^{t+1}\left(b_{k-1}\right)+\cdots+a_{k-1} \sigma^{k-1}(r) \sigma^{t+k-1}\left(b_{1}\right)=0$. 
Multiplying Eq.(2) by $\sigma^{t+k-1}\left(b_{1}\right) R$ on the left hand-side, we get $a_{k-1} R \sigma^{t}\left(b_{1}\right)=$ 0 for any nonnegative integer $t$, by the same arguments above. Continuing this process, we get $a_{i} R \sigma^{t}\left(b_{j}\right)=0$ for $i+j=k$ and any nonnegative integer $t$. Consequently, $a_{i} R \sigma^{t}\left(b_{j}\right)=0$ for any nonnegative integer $t$ and all $i, j$, entailing that $R$ satisfies the condition $(*)$.

Observe that the class of quasi $\sigma$-rigid rings does not depend on the class of $\sigma$-Armendariz rings each other. The quasi $\sigma$-rigid ring $R$, in [8, Example 1.1], is not $\sigma$-Armendariz for the automorphism $\sigma$ by [7, Example 13] and [10, Theorem 1.7]. Furthermore, the next example shows that there exists a $\sigma$-Armendariz ring which is not quasi $\sigma$-rigid for an automorphism $\sigma$ of $R$.

Example 2.3. Recall that for a $\operatorname{ring} R$ and an $(R, R)$-bimodule $M$, the trivial extension of $R$ by $M$ is the $\operatorname{ring} T(R, M)=R \oplus M$ with the usual addition and the following multiplication: $\left(r_{1}, m_{1}\right)\left(r_{2}, m_{2}\right)=\left(r_{1} r_{2}, r_{1} m_{2}+m_{1} r_{2}\right)$.

Let $R=T(\mathbb{Z}, \mathbb{Q})$ be the trivial extension of $\mathbb{Z}$ by $\mathbb{Q}$. Let $\sigma: R \rightarrow R$ be an automorphism defined by $\sigma((a, s))=(a, s / 2)$. Then $R$ is a $\sigma$-Armendariz ring by [10, Example 1.6]. However, $R$ is not quasi $\sigma$-rigid: Indeed, for $\left(\begin{array}{ll}0 & 1 \\ 0 & 0\end{array}\right) \neq 0$, we have $\left(\begin{array}{ll}0 & 1 \\ 0 & 0\end{array}\right)\left(\begin{array}{ll}a & t \\ 0 & a\end{array}\right) \sigma\left(\left(\begin{array}{ll}0 & 1 \\ 0 & 0\end{array}\right)\right)=0$ for any $a \in \mathbb{Z}$ and any $t \in \mathbb{Q}$. Note that it can be easily checked that $R$ is not semiprime, and $R[x ; \sigma]$ is not semiprime, either.

Based on these facts, we define the following that extends both $\sigma$-Armendariz rings and quasi $\sigma$-rigid rings, and that is an extension of quasi-Armendariz rings.

Definition 2.4. Let $\sigma$ be an endomorphism of a $\operatorname{ring} R$. The $\operatorname{ring} R$ is called a quasi-Armendariz ring with the endomorphism $\sigma$ (simply, a $\sigma$-quasi-Armendariz ring) if it satisfies the condition $(*)$.

Every $\sigma$-Armendariz ring is $\sigma$-quasi-Armendariz for an endomorphism $\sigma$ and every quasi $\sigma$-rigid ring is also $\sigma$-quasi-Armendariz for an automorphism $\sigma$ by Proposition 2.2; but the converses are not true by [8, Example 1.1] and Example 2.3 , respectively. Any quasi-Armendariz ring $R$ is an $\operatorname{id}_{R}$-quasi-Armendariz ring, where $\operatorname{id}_{R}$ is an identity endomorphism of $R$ and so every semiprime ring $R$ is $\operatorname{id}_{R}$-quasi-Armendariz by [5, Corollary 3.8].

Following [17], for an automorphism $\sigma$ of a $\operatorname{ring} R$, the $\operatorname{ring} R$ is called $\sigma$-semiprime if whenever $A$ is an ideal of $R$ and $m$ is an integer such that $A \sigma^{t}(A)=0$ for all $t \geq m$, then $A=0$. Notice that $R$ is a $\sigma$-semiprime ring if and only if the skew polynomial ring $R[x ; \sigma]$ is semiprime by $[17$, Proposition 1.1]. It is well-known that for an automorphism $\sigma$ of a $\operatorname{ring} R$, the $\operatorname{ring} R$ is $\sigma$-semiprime if and only if whenever $a \in R$ and $m$ is an integer such that $a R \sigma^{t}(a)=0$ for all $t \geq m$, then $a=0$. Quasi $\sigma$-rigid rings are clearly $\sigma$ semiprime.

Recall that $R$ is $\sigma$-rigid if and only if the skew polynomial ring $R[x ; \sigma]$ of $R$ is reduced if and only if $R$ is reduced and $\sigma$-Armendariz by [7, Proposition 3] 
and [10, Proposition 1.7]. Observe that there exists a semiprime ring $R$ with an automorphism $\sigma$ such that the skew polynomial $\operatorname{ring} R[x ; \sigma]$ of $R$ is not semiprime by Example 2.8 (below). However, we have the following:

Theorem 2.5. Let $\sigma$ be an endomorphism of a ring $R$.

(1) If $R$ is a semiprime and $\sigma$-quasi-Armendariz ring, then the skew polynomial ring $R[x ; \sigma]$ of $R$ is semiprime.

(2) If $R[x ; \sigma]$ is a semiprime ring, then $R$ is a $\sigma$-quasi-Armendariz ring.

(3) Let $\sigma$ be an automorphism of finite order. $R$ is a semiprime ring if and only if $R[x ; \sigma]$ is a semiprime ring.

Proof. (1) Let $R$ be a semiprime and $\sigma$-quasi-Armendariz ring. Assume that $p(x) R[x ; \sigma] p(x)=0$ where $p(x)=\sum_{i=0}^{m} a_{i} x^{i} \in R[x ; \sigma]$. Then $a_{i} R[x ; \sigma] a_{i}=0$, in particular $a_{i} R a_{i}=0$ for all $0 \leq i \leq m$. Since $R$ is semiprime, $a_{i}=0$ for all $0 \leq i \leq m$ and thus $p(x)=0$. Therefore $R[x ; \sigma]$ is semiprime.

(2) Let $R[x ; \sigma]$ be a semiprime ring. For any $a, b \in R$ and some nonnegative integer $l,\left(a x^{l}\right) R[x ; \sigma] b=0 \Leftrightarrow\left(b R[x ; \sigma]\left(a x^{l}\right) R[x ; \sigma]\right)^{2}=0 \Leftrightarrow b R[x ; \sigma] a=0 \Leftrightarrow$ $a R[x ; \sigma] b=0$, and we use this fact in the process. Suppose that $p(x)=$ $\sum_{i=0}^{m} a_{i} x^{i}, q(x)=\sum_{j=0}^{n} b_{j} x^{j} \in R[x ; \sigma]$ such that $p(x) R[x ; \sigma] q(x)=0$. We claim that $a_{i} R[x ; \sigma] b_{j}=0$ for all $0 \leq i \leq m$ and $0 \leq j \leq n$. When $i+j=0$, we can easily obtain that $a_{0} R[x ; \sigma] b_{0}=0$. Now we assume that our claim is true for $i+j \leq k-1$. From $p(x) R[x ; \sigma] q(x)=0$, we have

$$
a_{0}\left(r x^{t}\right) b_{k} x^{k}+a_{1} x\left(r x^{t}\right) b_{k-1} x^{k-1}+\cdots+a_{k} x^{k}\left(r x^{t}\right) b_{0}=0
$$

for any $r \in R$ and nonnegative integer $t$. Multiplying Eq.(3) by $b_{0} R[x ; \sigma]$ on the left hand-side, we get $b_{0} R[x ; \sigma] a_{k} x^{k}\left(r x^{t}\right) b_{0}=0$ by the induction hypothesis. Thus $\left(b_{0} R[x ; \sigma] a_{k} x^{k} R[x ; \sigma]\right)^{2}=0$ and so $b_{0} R[x ; \sigma] a_{k}=0$, an hence, $a_{k} R[x ; \sigma] b_{0}=0$ since $R[x ; \sigma]$ is semiprime. Then Eq.(3) becomes

$$
a_{0}\left(r x^{t}\right) b_{k} x^{k}+a_{1} x\left(r x^{t}\right) b_{k-1} x^{k-1}+\cdots+a_{k-1} x^{k-1}\left(r x^{t}\right) b_{1} x=0 .
$$

Multiplying Eq.(4) by $b_{1} x R[x ; \sigma]$ on the left hand-side, we similarly get

$$
b_{1} x R[x ; \sigma] a_{k-1} x^{k-1}\left(r x^{t}\right) b_{1} x=0,
$$

since $b_{1} x R[x ; \sigma] a_{i}=0$ for each $i \leq k-2$ by the induction hypothesis and the above arguments. Hence, $b_{1} x R[x ; \sigma] a_{k-1}=0$ and so $a_{k-1} R[x ; \sigma] b_{1}=$ 0 . Continuing this process, we have $a_{i} R[x ; \sigma] b_{j}=0$ for $i+j=k$. Thus $a_{i} R[x ; \sigma] b_{j}=0$ for any $0 \leq i \leq m$ and $0 \leq j \leq n$. Therefore $R$ is $\sigma$-quasiArmendariz.

(3) Let $\sigma^{u}=\operatorname{id}_{R}$ for some positive integer $u$. Assume that $R$ is a semiprime ring. First, we claim that $R$ is $\sigma$-quasi-Armendariz. Let $p(x)=\sum_{i=0}^{m} a_{i} x^{i}$ and $q(x)=\sum_{j=0}^{n} b_{j} x^{j} \in R[x ; \sigma]$ with $p(x) R[x ; \sigma] q(x)=0$. For any $r \in R$ and nonnegative integer $t$, we have

$$
\begin{aligned}
0 & =p(x) r x^{t} q(x) \\
& =a_{0} r \sigma^{t}\left(b_{0}\right) x^{t}+\left(a_{0} r \sigma^{t}\left(b_{1}\right)+a_{1} \sigma(r) \sigma^{t+1}\left(b_{0}\right)\right) x^{t+1}
\end{aligned}
$$




$$
+\cdots+a_{m} \sigma^{m}(r) \sigma^{t+m}\left(b_{n}\right) x^{m+n+t} .
$$

By the similar arguments to the proof of Proposition 2.2, we have $a_{i} R \sigma^{l}\left(b_{j}\right)=0$ for any $0 \leq i \leq m, 0 \leq j \leq n$ and $0 \leq l \leq u-1$, letting $\sigma^{t+s}(b)=\sigma^{l}(b)$ for any nonnegative integers $t$ and $s, 0 \leq l \leq u-1$ and $b \in R$. Hence, $R$ is $\sigma$-quasi-Armendariz. Consequently, $R[x ; \sigma]$ is a semiprime ring by (1).

Conversely, let $R[x ; \sigma]$ be a semiprime ring. Assume that $I$ is an ideal of $R$ with $I^{2}=0$. We claim that $I=0$. Let $J=I+\sigma(I)+\cdots+\sigma^{u-1}(I)$. Then $J$ is an ideal of $R$ and $\sigma(J) \subseteq J$, moreover $J[x ; \sigma]$ is an ideal of $R[x ; \sigma]$. Note that $J^{k}=0$ for some positive integer $k$ implies $J[x ; \sigma]=0$ : For, $(J[x ; \sigma])^{k} \subseteq$ $J \sigma^{i_{1}}(J) \cdots \sigma^{i_{k-1}}(J)[x ; \sigma] \subseteq J^{k}[x ; \sigma]=0$, since $\sigma^{i_{t}}(J) \subseteq J$ for any $i_{t} \geq 0$. Thus $J[x ; \sigma]=0$ since $R[x ; \sigma]$ is semiprime. Hence, from $J=I+\sigma(I)+\cdots+\sigma^{u-1}(I)$, $J^{u+1}=\left(I+\sigma(I)+\cdots+\sigma^{u-1}(I)\right)^{u+1}=\sum \sigma^{j_{1}}(I) \cdots \sigma^{j_{u+1}}(I)=0$ for $0 \leq$ $j_{1}, \ldots, j_{u+1} \leq u-1$ yields $J[x ; \sigma]=0$. Thus $J=0$, and so $I=0$. Therefore $R$ is semiprime.

Corollary 2.6. (1) [14, Theorem 10.19] $R$ is a semiprime ring if and only if so is the polynomial ring $R[x]$ over $R$.

(2) [5, Corollary 3.8] If $R$ is a semiprime ring, then $R$ is a quasi-Armendariz ring.

The class of semiprime rings and the class of $\sigma$-quasi-Armendariz rings do not depend on each other by Example 2.1 and Example 2.3. Notice that Example 2.3 illuminates that the condition " $R$ is a semiprime ring" in Theorem 2.5(1) is not superfluous (and hence shows that the converse of Theorem 2.5(2) is not true). The following example shows that the conclusion " $R[x ; \sigma]$ is semiprime" of Theorem 2.5(1) cannot be replaced by the condition " $R$ is a quasi $\sigma$-rigid ring".

Example 2.7. Let $R=\mathbb{Z}_{2} \oplus \mathbb{Z}_{2}$. Define $\sigma: R \rightarrow R$ by $\sigma((a, b))=(b, a)$. Then $R$ is a commutative reduced ring. Since $R$ is semiprime and $\sigma$ has an order 2, $R[x ; \sigma]$ is semiprime by Theorem $2.5(3)$. Thus $R$ is a $\sigma$-quasi-Armendariz ring by Theorem 2.5(2). However, $R$ is not quasi $\sigma$-rigid; indeed, $(1,0) R \sigma((1,0))=$ 0 but $(1,0) \neq 0$. Notice that $R$ is not $\sigma$-skew Armendariz (and hence, not $\sigma$-Armendariz) by [7, Example 2].

The following example shows that the condition " $R$ is a $\sigma$-quasi-Armendariz ring" in Theorem 2.5(1) cannot be dropped and that the condition " $\sigma$ has a finite order" in Theorem 2.5(3) is not superfluous.

Example 2.8. Let $F$ be a field and $F_{i}=F$ for $i \in \mathbb{Z}$. Let $R$ be a $F$-subalgebra of $\prod_{i \in \mathbb{Z}} F_{i}$ generated by $\oplus_{i \in \mathbb{Z}} F_{i}$ and $1_{\prod_{i \in \mathbb{Z}} F_{i}}$. Let $\sigma$ be an automorphism of $R$ defined by $\sigma\left(\left(a_{i}\right)\right)=\left(a_{i+1}\right)$. Then

$$
R=\left\{\left(a_{i}\right) \in \prod_{i \in \mathbb{Z}} F_{i} \mid a_{i} \text { is eventually constant }\right\}
$$


is reduced and von Neumann regular, but $R[x ; \sigma]$ is not semiprime by [11, Example 4.3]. Note that $R$ is not $\sigma$-quasi-Armendariz: In fact, let $p(x)=a x \in$ $R[x ; \sigma]$ where $a=(1,0,0, \ldots)$ then $p(x) R[x ; \sigma] p(x)=0$, but $a R a \neq 0$ and hence $a R[x ; \sigma] a \neq 0$.

Proposition 2.9. Let $\sigma$ be an epimorphism of a ring $R$.

(1) $R$ is a $\sigma$-quasi-Armendariz ring if and only if for every $p(x)=\sum_{i=0}^{m} a_{i} x^{i}$ and $q(x)=\sum_{j=0}^{n} b_{j} x^{j}$ in $R[x ; \sigma], p(x) R[x ; \sigma] q(x)=0$ implies $a_{0} R \sigma^{l}\left(b_{j}\right)=0$ for any nonnegative integer $l$ and $0 \leq j \leq n$.

(2) $R$ is a $\sigma$-skew quasi-Armendariz ring if and only if for every $p(x)=$ $\sum_{i=0}^{m} a_{i} x^{i}$ and $q(x)=\sum_{j=0}^{n} b_{j} x^{j}$ in $R[x ; \sigma], \quad p(x) R[x ; \sigma] q(x)=0$ implies $a_{0} R \sigma^{i}\left(b_{j}\right)=0$ for any $0 \leq i \leq m$ and $0 \leq j \leq n$.

Proof. (1) Assume that for every $p(x)=\sum_{i=0}^{m} a_{i} x^{i}$ and $q(x)=\sum_{j=0}^{n} b_{j} x^{j}$ in $R[x ; \sigma], p(x) R[x ; \sigma] q(x)=0$ implies $a_{0} R \sigma^{t}\left(b_{j}\right)=0$, equivalently $a_{0} R x^{t} b_{j}=0$ for any nonnegative integer $t$ and $0 \leq j \leq n$, we show that $R$ is a $\sigma$-quasiArmendariz ring. From $p(x) R[x ; \sigma] q(x)=0$ we get $p(x)\left(R x^{l}\right) q(x)=0$ for any nonnegative integer $l$, and hence $a_{0} R x^{l} q(x)=0$ by assumption. Hence, $0=\left(a_{0}+\cdots+a_{m} x^{m}\right) R x^{l} q(x)=\left(a_{1}+\cdots+a_{m} x^{m-1}\right) \sigma(R) x^{l}\left(\sigma\left(b_{0}\right)+\cdots+\sigma\left(b_{n}\right) x^{n}\right)$ yields $a_{1} R x^{l}\left(\sigma\left(b_{j}\right)\right)=0$ for each $0 \leq j \leq n$ by assumption. Inductively, we can see that $a_{i} R x^{l}\left(\sigma^{i}\left(b_{j}\right)\right)=0$ for any nonnegative integer $l, 0 \leq i \leq m$ and $0 \leq j \leq n$. Consequently, $a_{i} R \sigma^{t}\left(b_{j}\right)=0$ for any nonnegative integer $t$, showing that $R$ is a $\sigma$-quasi-Armendariz ring. The converse is clear.

(2) can be shown by the same arguments as in the proof of (1), letting $l=0$.

Corollary 2.10. $R$ is a quasi-Armendariz ring if and only if for every polynomials $f(x)=\sum_{i=0}^{m} a_{i} x^{i}$ and $g(x)=\sum_{j=0}^{n} b_{j} x^{j}$ in $R[x], f(x) R[x] g(x)=0$ implies $a_{0} R b_{j}=0$ for each $0 \leq j \leq n$.

Proposition 2.9 says that every $\sigma$-quasi-Armendariz ring is $\sigma$-skew quasiArmendariz for an epimorphism $\sigma$, and so one may ask whether the converse does hold. However the answer is negative by Example 2.8: In fact, the ring $R$ in Example 2.8 is $\sigma$-skew quasi-Armendariz by [9, Example 1.8]. Moreover, Example 2.8 shows that there exists a reduced and von Neumann regular ring with the automorphism $\sigma$ which is not $\sigma$-quasi-Armendariz, and hence not $\sigma$-rigid.

Recall that an endomorphism $\sigma$ of a ring $R$ is called semicommutative [2, Definition 2.1] if whenever $a b=0$ for $a, b \in R, a R \sigma(b)=0$; a $\operatorname{ring} R$ is called $\sigma$-semicommutative if there exists a semicommutative endomorphism $\sigma$ of $R$. Note that $R$ is a reduced and $\sigma$-semicommutative ring for a monomorphism $\sigma$ if and only if $R$ is a $\sigma$-rigid ring by [2, Theorem 2.4]. The semiprimeness and the $\sigma$-semicommutativity of a ring are independent of each other by $[2$, Example 2.3 and Example 2.5(1)]. 
Proposition 2.11. Let $\sigma$ be an automorphism of a ring $R$. Assume that $R$ is a $\sigma$-semicommutative and semiprime ring. Then the following are equivalent:

(1) $R$ is $\sigma$-rigid.

(2) $R$ is quasi $\sigma$-rigid.

(3) $R$ is $\sigma$-semiprime.

(4) $R$ is $\sigma$-Armendariz.

(5) $R$ is $\sigma$-skew Armendariz.

(6) $R$ is $\sigma$-quasi-Armendariz.

(7) $R$ is $\sigma$-skew quasi-Armendariz.

Proof. $(1) \Leftrightarrow(2) \Leftrightarrow(3)$ are shown in [8, Proposition 3.4] when $R$ is $\sigma$-semicommutative. esis.

$(1) \Rightarrow(4) \Rightarrow(5)$ By $[6$, Proposition 6] and [10, Theorem 1.8] without hypoth-

$(5) \Rightarrow(7)$ is well-known when $\sigma$ is an epimorphism.

$(7) \Rightarrow(6)$ can be shown with the help of that $R$ is $\sigma$-semicommutative.

$(6) \Rightarrow(1)$ Suppose that $R$ is $\sigma$-quasi-Armendariz. Let $a \sigma(a)=0$ for $a \in R$. Then we get $a R \sigma^{k}(a)=0$ for any $k \geq 2$, since $R$ is $\sigma$-semicommutative. Put $p(x)=a x^{2}$. Then $p(x) R[x ; \sigma] a=0$, and so $a R[x ; \sigma] a=0$ and $a R a=0$, entailing that $a=0$ since $R$ is semiprime. Thus $R$ is $\sigma$-rigid.

The following gives us basic examples for $\sigma$-skew quasi-Armendariz rings.

Theorem 2.12. For an endomorphism $\sigma$ of a ring $R$, let $p(x)=\sum_{i=0}^{m} a_{i} x^{i}$ and $q(x)=\sum_{j=0}^{n} b_{j} x^{j} \in R[x ; \sigma]$.

(1) If $R$ is a reduced ring, then $p(x) R[x ; \sigma] q(x)=0$ implies $a_{i} \sigma^{i}\left(b_{j}\right)=0$ for all $i$ and $j$.

(2) If the skew polynomial ring $R[x ; \sigma]$ of $R$ is quasi-Armendariz, then $p(x) R[x ; \sigma] q(x)=0$ implies $a_{i} x^{i} R b_{j} x^{j}=0$ for all $i$ and $j$.

Proof. (1) Let $R$ be a reduced ring. From $p(x) R[x ; \sigma] q(x)=0$, we get $p(x) r q(x)$ $=\left(a_{0}+a_{1} x+\cdots+a_{m} x^{m}\right)\left(r b_{0}+r b_{1} x+\cdots+r b_{n} x^{n}\right)=0$ for any $r \in R$. We claim that $a_{i} \sigma^{i}\left(b_{j}\right)=0$ for all $i$ and $j$. We proceed by induction on $i+j$. If $i+j=0$, then $a_{0} R b_{0}=0$ and so $a_{0} b_{0}=0$. Assume that we have $a_{i} \sigma^{i}\left(b_{j}\right)=0$ for $i+j \leq k-1$. Then for any $r \in R$,

(5) $a_{0} r b_{k}+a_{1} \sigma(r) \sigma\left(b_{k-1}\right)+\cdots+a_{k-1} \sigma^{k-1}(r) \sigma^{k-1}\left(b_{1}\right)+a_{k} \sigma^{k}(r) \sigma^{k}\left(b_{0}\right)=0$.

Letting $r=b_{0}$ in Eq.(5), we have $a_{k} \sigma^{k}\left(b_{0}\right) \sigma^{k}\left(b_{0}\right)=0$, by the induction hypothesis. Since $R$ is reduced, $a_{k} \sigma^{k}\left(b_{0}\right)=0$, equivalently, $a_{k} R \sigma^{k}\left(b_{0}\right)=0$. Eq.(5) becomes

$$
a_{0} r b_{k}+a_{1} \sigma(r) \sigma\left(b_{k-1}\right)+\cdots+a_{k-1} \sigma^{k-1}(r) \sigma^{k-1}\left(b_{1}\right)=0 .
$$

Letting $r=b_{1}$ in Eq.(6), we have $a_{k-1} \sigma^{k-1}\left(b_{1}\right)=0$, and so $a_{k-1} R \sigma^{k-1}\left(b_{1}\right)=0$ by the same method as above. Continuing this process, we get $a_{i} \sigma^{i}\left(b_{j}\right)=0$ for $i+j=k$, consequently, $a_{i} \sigma^{i}\left(b_{j}\right)=0$ for all $0 \leq i \leq m$ and $0 \leq j \leq n$. 
(2) Assume that $R[x ; \sigma]$ is a quasi-Armendariz ring. Let $p(x) R[x ; \sigma] q(x)=0$. Then for any $r \in R$ and nonnegative integer $t$,

(7)

$$
a_{0} r \sigma^{t}\left(b_{k}\right)+a_{1} \sigma(r) \sigma^{t+1}\left(b_{k-1}\right)+\cdots+a_{k-1} \sigma^{k-1}(r) \sigma^{k+t-1}\left(b_{1}\right)+a_{k} \sigma^{k}(r) \sigma^{k+t}\left(b_{0}\right)=0,
$$

where $0 \leq k \leq m+n$. Set $f(y)=a_{0}+\left(a_{1} x\right) y+\cdots+\left(a_{m} x^{m}\right) y^{m}$ and $g(y)=b_{0}+$ $\left(b_{1} x\right) y+\cdots+\left(b_{n} x^{n}\right) y^{n}$ in $(R[x ; \sigma])[y]$. This proves that $f(y)(R[x ; \sigma])[y] g(y)=0$ holds. Since $R[x ; \sigma]$ is quasi-Armendariz, we obtain $a_{i} x^{i} R[x ; \sigma] b_{j} x^{j}=0$, and so $a_{i} x^{i} R b_{j} x^{j}=0$ for $0 \leq i \leq m$ and $0 \leq j \leq n$.

From Theorem 2.12, we obtain:

Corollary 2.13. For an endomorphism $\sigma$ of a ring $R$, the ring $R$ is $\sigma$-skew quasi-Armendariz, if either $R$ is a reduced ring or $R[x ; \sigma]$ is a quasi-Armendariz ring with an epimorphism $\sigma$.

For an endomorphism $\sigma$ and an ideal $I$ of a $\operatorname{ring} R, I$ is called a $\sigma$-ideal if $\sigma(I) \subseteq I$.

Proposition 2.14. For an endomorphism $\sigma$ of a ring $R$, we have the following.

(1) Let $\left\{I_{\gamma} \mid \gamma \in \Gamma\right\}$ be a family of $\sigma$-ideals of $R$. If $R$ is a subdirect sum of $\sigma$-quasi-Armendariz rings, then $R$ is a $\sigma$-quasi-Armendariz ring.

(2) If $S$ is a ring and $\alpha: R \rightarrow S$ is a ring isomorphism, then, $R$ is a $\sigma$-quasi-Armendariz ring if and only if $S$ is an $\alpha \sigma \alpha^{-1}$-quasi-Armendariz ring.

Proof. (1) Observe that $\cap_{\gamma \in \Gamma} I_{\gamma}=0$. Let $p(x)=\sum_{i=0}^{m} a_{i} x^{i}$ and $q(x)=$ $\sum_{j=0}^{n} b_{j} x^{j}$ in $R[x ; \sigma]$ with $p(x) R[x ; \sigma] q(x)=0$. Since $R / I_{\gamma}$ is $\sigma$-quasi-Armendariz for any $\gamma \in \Gamma, a_{i} R \sigma^{t}\left(b_{j}\right) \subseteq I_{\gamma}$ for all $i, j$ and nonnegative integer $t$, and so $a_{i} R \sigma^{t}\left(b_{j}\right)=0$. Therefore $R$ is $\sigma$-quasi-Armendariz.

(2) For $a \in R$, let $a^{\prime}=\alpha(a)$. Note that $p(x)=\sum_{i=0}^{m} a_{i} x^{i}$ and $q(x)=$ $\sum_{j=0}^{n} b_{j} x^{j}$ in $R[x ; \sigma]$ if and only if $p^{\prime}(x)=\sum_{i=0}^{m} a_{i}^{\prime} x^{i}$ and $q^{\prime}(x)=\sum_{j=0}^{n} b_{j}^{\prime} x^{j}$ in $S\left[x ; \alpha \sigma \alpha^{-1}\right]$. Also, for any $r \in R$ and nonnegative integer $t, p(x) r x^{t} q(x)=0$ if and only if $\sum_{i+j=k} a_{i} \sigma^{i}(r) \sigma^{i+t}\left(b_{j}\right)=0$ for each $0 \leq k \leq m+n$ if and only if $\sum_{i+j=k} \alpha\left(a_{i}\right)\left(\alpha \sigma \alpha^{-1}\right)^{i}(\alpha(r))\left(\alpha \sigma \alpha^{-1}\right)^{i+t}\left(\alpha\left(b_{j}\right)\right)=0$ for each $0 \leq k \leq$ $m+n$, since $\left(\alpha \sigma \alpha^{-1}\right)^{w}=\alpha \sigma^{w} \alpha^{-1}$ for any positive integer $w$. Equivalently, for any $s \in S$ and nonnegative integer $t, \sum_{i+j=k} a_{i}^{\prime}\left(\alpha \sigma \alpha^{-1}\right)^{i}(s)\left(\alpha \sigma \alpha^{-1}\right)^{i+t}\left(b_{j}^{\prime}\right)=$ 0 for each $0 \leq k \leq m+n$ if and only if $p^{\prime}(x) s x^{t} q^{\prime}(x)=0$ if and only if $p^{\prime}(x) S\left[x ; \alpha \sigma \alpha^{-1}\right] q^{\prime}(x)=0$. Hence, for all $i, j$ and any nonnegative integer $t$, $a_{i} R \sigma^{t}\left(b_{j}\right)=0$ if and only if $\alpha\left(a_{i}\right) S \alpha \sigma^{t}\left(b_{j}\right)=0$ if and only if $a_{i}^{\prime} S\left(\alpha \sigma \alpha^{-1}\right)^{t}\left(b_{j}^{\prime}\right)=$ 0 . The proof is completed.

Corollary 2.15 ([5, Proposition 3.7]). If $R$ is a subdirect sum of quasi-Armendariz rings, then $R$ is a quasi-Armendariz ring. 


\section{Examples of $\sigma$-quasi-Armendariz rings}

Hirano proved that the $n \times n$ full (or upper triangular) matrix ring over a quasi-Armendariz ring is quasi-Armendariz [5, Theorem 3.12 and Corollary 3.15]. We extend these results to $\sigma$-quasi-Armendariz rings.

Recall that if $\sigma$ is an endomorphism of a ring $R$, then $\sigma$ can be extended to the endomorphism $\bar{\sigma}$ of $\operatorname{Mat}_{n}(R)$ over $R$ defined by $\bar{\sigma}\left(\left(a_{i j}\right)\right)=\left(\sigma\left(a_{i j}\right)\right)$.

Theorem 3.1. For an endomorphism $\sigma$ of a ring $R$, the following are equivalent:

(1) $R$ is a $\sigma$-quasi-Armendariz ring.

(2) $\operatorname{Mat}_{n}(R)$ is a $\bar{\sigma}$-quasi-Armendariz ring for any $n \geq 2$.

(3) $\operatorname{Mat}_{n}(R)$ is a $\bar{\sigma}$-quasi-Armendariz ring for some $n \geq 2$.

Proof. $(1) \Rightarrow(2)$ Let $R$ be a $\sigma$-quasi-Armendariz ring. Note that $\operatorname{Mat}_{n}(R)[x ; \bar{\sigma}]$ $\cong \operatorname{Mat}_{n}(R[x ; \sigma])$. Let $p(x)=\sum_{i=0}^{l} A_{i} x^{i}, q(x)=\sum_{j=0}^{m} B_{j} x^{j} \in \operatorname{Mat}_{n}(R)[x ; \sigma]$ with $A_{i}=\left(a_{s t}^{i}\right)$ and $B_{j}=\left(b_{v w}^{j}\right)$. We can write

$$
p(x)=\left(p_{s t}\right), q(x)=\left(q_{v w}\right) \in \operatorname{Mat}_{n}(R[x ; \sigma]) \text { with } p_{s t}=\sum_{i=0}^{l} a_{s t}^{i} x^{i}, q_{v w}=\sum_{j=0}^{m} b_{v w}^{j} x^{j} .
$$

Put $p(x) \operatorname{Mat}_{n}(R)[x ; \bar{\sigma}] q(x)=0$, then equivalently $p(x) \operatorname{Mat}_{n}(R[x ; \sigma]) q(x)=0$. Let $E_{i j}$ 's be the matrix units of $\operatorname{Mat}_{n}(R)$ with $(i, j)$-entry 1 and zero elsewhere. From $p(x)\left(R E_{h k} x^{t}\right) g(x)=0$ for any nonnegative integer $t$, we get

$$
p_{\alpha h}\left(r x^{t}\right) q_{k \beta}=0 \text { for any } r \in R \text { and all } 1 \leq \alpha, \beta \leq n .
$$

Since $R$ is $\sigma$-quasi-Armendariz, we have $a_{s t}^{i}\left(r x^{t}\right) b_{v w}^{j}=0$ for any $r \in R$ and nonnegative integer $t$ and all $0 \leq i \leq l, 0 \leq j \leq m$ and $1 \leq s, t, v, w \leq n$. It then follows that

$$
A_{i} \operatorname{Mat}_{n}(R[x ; \sigma]) B_{j}=0 \text { for all } 0 \leq i \leq l \text { and } 0 \leq j \leq m,
$$

concluding that $\operatorname{Mat}_{n}(R)$ is $\bar{\sigma}$-quasi-Armendariz.

$(2) \Rightarrow(3)$ is obvious.

$(3) \Rightarrow(1)$ Suppose that $\operatorname{Mat}_{w}(R)$ is $\bar{\sigma}$-quasi-Armendariz for some $w \geq 2$. Let $p(x) R[x ; \sigma] q(x)=0$ with $p(x)=\sum_{i=0}^{m} a_{i} x^{i}$ and $q(x)=\sum_{j=0}^{n} b_{j} x^{j}$ in $R[x ; \sigma]$. Then

$$
\left(p(x) \sum_{k=1}^{w} E_{k k}\right) \operatorname{Mat}_{w}(R[x ; \sigma])\left(q(x) \sum_{k=1}^{w} E_{k k}\right)=0 .
$$

Since $\operatorname{Mat}_{w}(R)$ is $\bar{\sigma}$-quasi-Armendariz, we have

$$
\left(a_{i} \sum_{k=1}^{w} E_{k k}\right)\left(\operatorname{Mat}_{w}(R) x^{t}\right)\left(b_{j} \sum_{k=1}^{w} E_{k k}\right)=0
$$

for any nonnegative integer $t$ and all $i$ and $j$; in particular,

$$
\left(a_{i} \sum_{k=1}^{w} E_{k k}\right)\left(r x^{t} \sum_{k=1}^{w} E_{k k}\right)\left(b_{j} \sum_{k=1}^{w} E_{k k}\right)=0
$$


for any $r \in R$, obtaining $a_{i}\left(R x^{t}\right) b_{j}=0$. Therefore $R$ is $\sigma$-quasi-Armendariz.

Observe that we obtain the following result for $U_{n}(R)$ over a $\sigma$-quasi-Armendariz ring $R$, by the same method as in the proof of Theorem 3.1.

Theorem 3.2. For an endomorphism $\sigma$ of a ring $R$, the following are equivalent:

(1) $R$ is $\sigma$-quasi-Armendariz.

(2) $U_{n}(R)$ is $\bar{\sigma}$-quasi-Armendariz for any $n \geq 2$.

(3) $U_{n}(R)$ is $\bar{\sigma}$-quasi-Armendariz for some $n \geq 2$.

Corollary 3.3 ([5, Corollary 3.15]). If $R$ is a quasi-Armendariz, then for any positive integer $n, U_{n}(R)$ is also a quasi-Armendariz ring.

For a ring $R$ and $n \geq 2$, let

$$
\begin{gathered}
S_{n}(R)=\left\{\left(\begin{array}{ccccc}
a & a_{12} & a_{13} & \cdots & a_{1 n} \\
0 & a & a_{23} & \cdots & a_{2 n} \\
0 & 0 & a & \cdots & a_{3 n} \\
\vdots & \vdots & \vdots & \ddots & \vdots \\
0 & 0 & 0 & \cdots & a
\end{array}\right) \mid a, a_{i j} \in R\right\} \text { and } \\
V_{n}(R)=\left\{\left(\begin{array}{ccccc}
a_{1} & a_{2} & a_{3} & \cdots & a_{n} \\
0 & a_{1} & a_{2} & \cdots & a_{n-1} \\
\vdots & \vdots & \vdots & \vdots & \vdots \\
0 & 0 & 0 & \cdots & a_{2} \\
0 & 0 & 0 & \cdots & a_{1}
\end{array}\right) \mid a_{1}, \ldots, a_{n} \in R\right\} .
\end{gathered}
$$

Related to Theorem 3.1 and Theorem 3.2, one may suspect that $S_{n}(R)$ and $V_{n}(R)$ may be also $\bar{\sigma}$-quasi-Armendariz rings for any $n \geq 2$, where $R$ is a $\sigma$ quasi-Armendariz ring with an endomorphism $\sigma$. But the possibility is erased by the next example, and so the subring of a $\sigma$-quasi-Armendariz ring need not to be $\sigma$-quasi-Armendariz:

Example 3.4. Let $W$ be an $\operatorname{id}_{W}$-rigid (i.e., reduced) ring where $\operatorname{id}_{W}$ is the identity endomorphism of a ring $W$. Then the trivial extension $R=T(W, W)$ of $W$ is an $\mathrm{id}_{R}$-Armendariz ring by [10, Corollary 2.2], and thus $R$ is id $R^{\text {-quasi- }}$ Armendariz. Then it can be proved that $S_{n}(R)\left(V_{n}(R)\right)$ is not $\overline{\mathrm{id}}_{S_{n}(R)}\left(\overline{\mathrm{id}}_{V_{n}(R)}\right)$ quasi-Armendariz for all $n \geq 2$, with the help of [3, Example 2.5].

By [10, Proposition 2.1 and Corollary 2.2], if $R$ is a $\sigma$-rigid ring, then $S_{2}(R)$ and $S_{3}(R)$ are $\bar{\sigma}$-Armendariz rings, and so they are $\bar{\sigma}$-quasi-Armendariz for an endomorphism $\sigma$ of $R$; while $S_{n}(R)$ is not $\bar{\sigma}$-Armendariz for $n \geq 4$ by [10, Theorem 1.8] and [7, Example 18], even if $R$ is a $\sigma$-rigid ring. However, we have the following.

Lemma 3.5 ([3, Lemma 2.6]). A ring $R$ is semiprime if and only if $a R b=0$ for $a, b \in R$ implies $a R \cap R b=0$. 
Theorem 3.6. Let $\sigma$ be an endomorphism of a ring $R$.

(1) Assume that the skew polynomial $R[x ; \sigma]$ of $R$ is a semiprime ring. Then $S_{n}(R)$ and $V_{n}(R)$ are $\bar{\sigma}$-quasi-Armendariz rings for any $n \geq 2$.

(2) If $V_{n}(R)$ (or, $\left.S_{n}(R)\right)$ is a $\bar{\sigma}$-quasi-Armendariz ring for $n \geq 2$, then $R$ is a $\sigma$-quasi-Armendariz ring.

Proof. (1) Note that $S_{n}(R)[x ; \bar{\sigma}] \cong S_{n}(R[x ; \sigma])$ for $n \geq 2$. Then every polynomial $p(x)=\sum_{u=0}^{m} A_{u} x^{u} \in S_{n}(R)[x ; \bar{\sigma}]$ can be expressed by the form of

$$
\left(\begin{array}{ccccc}
p_{11} & p_{12} & p_{13} & \cdots & p_{1 n} \\
0 & p_{11} & p_{23} & \cdots & p_{2 n} \\
0 & 0 & p_{11} & \cdots & p_{3 n} \\
\vdots & \vdots & \vdots & \ddots & \vdots \\
0 & 0 & 0 & \cdots & p_{11}
\end{array}\right)=\left(p_{11}, p_{12}, \ldots, p_{(n-1) n}\right)
$$

where $A_{u}=\left(a_{i j}^{u}\right) \in S_{n}(R)$ for any $0 \leq u \leq m$ and $p_{i j}=\sum_{u=0}^{m} a_{i j}^{u} x^{u} \in$ $R[x ; \sigma]$ for any $1 \leq i, j \leq n$. Assume $p(x) S_{w}(R)[x ; \bar{\sigma}] q(x)=0$ for $w \geq 2$, where $p(x)=\sum_{u=0}^{m} A_{u} x^{u}=\left(p_{11}, p_{12}, \ldots, p_{(w-1) w}\right)$ and $q(x)=\sum_{v=0}^{n} B_{v} x^{v}=$ $\left(q_{11}, q_{12}, \ldots, q_{(w-1) w}\right) \in S_{w}(R)[x ; \bar{\sigma}], A_{u}=\left(a_{i j}^{u}\right), B_{v}=\left(b_{s t}^{v}\right) \in S_{w}(R)$ for any $0 \leq u \leq m, 0 \leq v \leq n$ and $p_{i j}, q_{s t} \in R[x ; \sigma]$ for any $1 \leq i, j, s, t \leq w$. We claim that $A_{u} S_{w}(R[x ; \sigma]) B_{v}=0$ for any $0 \leq u \leq m$, and $0 \leq v \leq n$. We proceed by induction on $w$. For $w=2$, suppose that $p(x) S_{2}(R)[x ; \bar{\sigma}] q(x)=0$ with $p(x)=$ $\left(p_{11}, p_{12}\right), q(x)=\left(q_{11}, q_{12}\right) \in S_{2}(R)[x ; \bar{\sigma}]$. Then $\left(p_{11}, p_{12}\right)\left(r_{11} x^{l}, r_{12} x^{l}\right)\left(q_{11}, q_{12}\right)$ $=0$ for any $r_{11}, r_{12} \in R$ and nonnegative integer $l$, and so we have

$$
\begin{gathered}
p_{11}\left(r_{11} x^{l}\right) q_{11}=0, \\
p_{11}\left(r_{11} x^{l}\right) q_{12}+p_{11}\left(r_{12} x^{l}\right) q_{11}+p_{12}\left(r_{11} x^{l}\right) q_{11}=0 .
\end{gathered}
$$

From Eq.(8), $p_{11} R[x ; \sigma] q_{11}=0$ and hence $a_{11}^{u} R[x ; \sigma] b_{11}^{v}=0$ for all $0 \leq u \leq m$ and $0 \leq v \leq n$ since $R$ is $\sigma$-quasi-Armendariz by Theorem 2.5(2). Then Eq.(9) becomes

$$
p_{11}\left(r_{11} x^{l}\right) q_{12}+p_{12}\left(r_{11} x^{l}\right) q_{11}=0 .
$$

Since $p_{11} R[x ; \sigma] q_{11}=0$, we get $p_{11}\left(r_{11} x^{l}\right) q_{12}=-p_{12}\left(r_{11} x^{l}\right) q_{11} \in p_{11} R[x ; \sigma] \cap$ $R[x ; \sigma] q_{11}=0$ by Lemma 3.5 , and so $p_{11} R[x ; \sigma] q_{12}=0$ and $p_{12} R[x ; \sigma] q_{11}=0$. Thus $a_{11}^{u} R[x ; \sigma] b_{12}^{v}=0$ and $a_{12}^{u} R[x ; \sigma] b_{11}^{v}=0$ for all $0 \leq u \leq m$ and $0 \leq v \leq n$, since $R$ is $\sigma$-quasi-Armendariz. These imply that $A_{u} S_{2}(R[x ; \sigma]) B_{v}=0$ for all $0 \leq u \leq m$ and $0 \leq v \leq n$, and therefore $S_{2}(R)$ is $\bar{\sigma}$-quasi-Armendariz. Assume that our claim is true for $2 \leq w \leq k-1$. Let $p(x) S_{k}(R)[x ; \bar{\sigma}] q(x)=0$ with $p(x)=\left(p_{11}, p_{12}, \ldots, p_{(k-1) k}\right)$ and $q(x)=\left(q_{11}, q_{12}, \ldots, q_{(k-1) k}\right) \in S_{k}(R)[x ; \bar{\sigma}]$. Then for any nonnegative integer $l$ and $r_{11}, r_{12}, \ldots, r_{(k-1) k} \in R$,

$$
\left(p_{11}, p_{12}, \ldots, p_{(k-1) k}\right)\left(r_{11} x^{l}, r_{12} x^{l}, \ldots, r_{(k-1) k} x^{l}\right)\left(q_{11}, q_{12}, \ldots, q_{(k-1) k}\right)=0 .
$$

By the induction hypothesis, we have $p_{i j} R[x ; \sigma] q_{s t}=0$ and so $a_{i j}^{u} R[x ; \sigma] b_{s t}^{v}=0$ for all $0 \leq u \leq m, 0 \leq v \leq n$ and $1 \leq i, j, s, t \leq k-1$. Hence, from Eq.(11) we 
have the following equations:

$(\mathbf{1}, \mathbf{k}) p_{11}\left(r_{11} x^{l}\right) q_{1 k}+\left[p_{11}\left(r_{12} x^{l}\right)+p_{12}\left(r_{11} x^{l}\right)\right] q_{2 k}+\cdots+\left[p_{11}\left(r_{1 k} x^{l}\right)+p_{12}\left(r_{2 k} x^{l}\right)+\right.$ $\left.\cdots+p_{1 k}\left(r_{11} x^{l}\right)\right] q_{11}=0$

$(\mathbf{2}, \mathbf{k}) p_{11}\left(r_{11} x^{l}\right) q_{2 k}+\left[p_{11}\left(r_{23} x^{l}\right)+p_{23}\left(r_{11} x^{l}\right)\right] q_{3 k}+\cdots+\left[p_{11}\left(r_{2 k} x^{l}\right)+p_{23}\left(r_{3 k} x^{l}\right)+\right.$ $\left.\cdots+p_{2 k}\left(r_{11} x^{l}\right)\right] q_{11}=0$,

(k-2,k) $p_{11}\left(r_{11} x^{l}\right) q_{(k-2) k}+\left[p_{11}\left(r_{(k-2)(k-1)} x^{l}\right)+p_{(k-1)(k-1)}\left(r_{11} x^{l}\right)\right] q_{(k-1) k}+$ $\left[p_{11}\left(r_{(k-2) k} x^{l}\right)+p_{(k-1)(k-1)}\left(r_{(k-1) k} x^{l}\right)+p_{(k-2) k}\left(r_{11} x^{l}\right)\right] q_{11}=0$,

$\left(\mathbf{k - 1 , k )} p_{11}\left(r_{11} x^{l}\right) q_{(k-1) k}+\left[p_{11}\left(r_{(k-1) k} x^{l}\right)+p_{(k-1) k}\left(r_{11} x^{l}\right)\right] q_{11}=0\right.$.

Since $p_{11} R[x ; \sigma] q_{11}=0$, we obtain $p_{11}\left(r_{11} x^{l}\right) q_{(k-1) k}+p_{(k-1) k}\left(r_{11} x^{l}\right) q_{11}=0$ from $(\mathrm{k}-1, \mathrm{k})$, and so

$$
p_{11}\left(r_{11} x^{l}\right) q_{(k-1) k}=-p_{(k-1) k}\left(r_{11} x^{l}\right) q_{11} \in p_{11} R[x ; \sigma] \cap R[x ; \sigma] q_{11}=0
$$

by Lemma 3.5. Thus

$$
p_{11} R[x ; \sigma] q_{(k-1) k}=0 \text { and } p_{(k-1) k} R[x ; \sigma] q_{11}=0 .
$$

By Eq.(12) and the induction hypothesis, $(\mathbf{k - 2 , k})$ becomes $p_{11}\left(r_{11} x^{l}\right) q_{(k-2) k}+$ $p_{(k-1)(k-1)}\left(r_{11} x^{l}\right) q_{(k-1) k}+p_{(k-2) k}\left(r_{11} x^{l}\right) q_{11}=0$, and so

(13) $\quad p_{11} R[x ; \sigma] q_{(k-2) k}+p_{(k-1)(k-1)} R[x ; \sigma] q_{(k-1) k}+p_{(k-2) k} R[x ; \sigma] q_{11}=0$.

Multiplying Eq.(13) by $q_{11} R[x ; \sigma]$ on the left hand-side, we similarly get

$$
q_{11} R[x ; \sigma] p_{(k-2) k} R[x ; \sigma] q_{11}=0,
$$

and hence $p_{(k-2) k} R[x ; \sigma] q_{11}=0$ and thus

$p_{11} R[x ; \sigma] q_{(k-2) k}=-p_{(k-1)(k-1)} R[x ; \sigma] q_{(k-1) k} \subseteq p_{11} R[x ; \sigma] \cap R[x ; \sigma] q_{(k-1) k}=0$

by the induction hypothesis and the above arguments. Then we have

$$
p_{11} R[x ; \sigma] q_{(k-2) k}=0
$$

and $p_{(k-1)(k-1)} R[x ; \sigma] q_{(k-1) k}=0$. Continuing this procedure yields

$$
p_{i j} R[x ; \sigma] q_{s t}=0
$$

for any $1 \leq i, j, s, t \leq k$. Consequently, $a_{i j}^{u} R[x ; \sigma] b_{s t}^{v}=0$ for any $1 \leq i, j, s, t \leq$ $k, 0 \leq u \leq m$ and $0 \leq v \leq n$. Thus $A_{u} S_{k}(R)[x ; \bar{\sigma}] B_{v}=A_{u} S_{k}(R[x ; \sigma]) B_{v}=\bar{O}$ for any $0 \leq u \leq m$ and $0 \leq v \leq n$. Therefore $S_{w}(R)$ is $\bar{\sigma}$-quasi-Armendariz for any $w \geq 2$. Similarly, it is shown that $V_{n}(R)$ is $\bar{\sigma}$-quasi-Armendariz for any $n \geq 2$. 3.1 .

(2) is proved by the same arguments as in the proof of $(3) \Rightarrow(1)$ of Theorem

In general, $S_{n}(R)$ and $V_{n}(R)$ for $n \geq 2$ are not semiprime rings, even if $R$ is a semiprime ring. But we get the following by [14, Theorem 10.19] and Theorem 3.6 . 
Corollary 3.7. If $R$ is a semiprime ring, then $S_{n}(R)$ and $V_{n}(R)$ for any $n \geq 2$ are quasi-Armendariz rings. If $S_{n}(R)$ (or, $\left.V_{n}(R)\right)$ for $n \geq 2$ is a quasiArmendariz ring, then $R$ is a quasi-Armendariz ring.

For an endomorphism $\sigma$ and a $\sigma$-ideal $I$ of a $\operatorname{ring} R, \bar{\sigma}: R / I \rightarrow R / I$ defined by $\bar{\sigma}(a+I)=\sigma(a)+I$ for $a \in R$ is an endomorphism of the factor ring $R / I$. Note that $V_{n}(R) \cong R[x] /\left\langle x^{n}\right\rangle$ by [15], where $\left\langle x^{n}\right\rangle$ is an ideal of the polynomial ring $R[x]$ over $R$ generated by $x^{n}$. The next corollary follows directly from Theorem 3.6.

Corollary 3.8. Let $\sigma$ be an endomorphism of a ring $R$. If the skew polynomial ring $R[x ; \sigma]$ of $R$ is a semiprime ring, then the factor ring $R[x] /\left\langle x^{n}\right\rangle$ is $\bar{\sigma}$-quasiArmendariz for $n \geq 2$.

The following example shows that the homomorphic image of a $\sigma$-quasiArmendariz ring may not necessarily be $\bar{\sigma}$-quasi-Armendariz.

Example 3.9. Let $R=T\left(\mathbb{Z}, \mathbb{Z}_{4}\right)$ be the trivial extension of $\mathbb{Z}$ by $\mathbb{Z}_{4}$, and $\sigma: R \rightarrow R$ be defined by $\sigma((a, \bar{s}))=(a,-\bar{s})$. Then $R$ is $\sigma$-Armendariz by [10, Example 1.10], and so $R$ is $\sigma$-quasi-Armendariz. However, for a $\sigma$-ideal $I=\{(a, \overline{0}) \mid a \in 4 \mathbb{Z}\}$ of $R$, the factor $\operatorname{ring} R / I \cong\left\{(\bar{a}, \bar{b}) \mid \bar{a}, \bar{b} \in \mathbb{Z}_{4}\right\}$ is not $\bar{\sigma}$-quasi-Armendariz: Indeed, $((\overline{2}, \overline{0})+(\overline{2}, \overline{1}) x)(R / I)[x ; \bar{\sigma}]((\overline{2}, \overline{0})+(\overline{2}, \overline{1}) x)=0$, but $0 \neq(\overline{2}, \overline{0})(\overline{1}, \overline{0})(\overline{2}, \overline{1}) \in(\overline{2}, \overline{0})(R / I)(\overline{2}, \overline{1})$, and so $(\overline{2}, \overline{0})(R / I)[x ; \bar{\sigma}](\overline{2}, \overline{1}) \neq 0$.

For a nonempty subset $S$ of a ring $R$, we write $r_{R}(S)=\{c \in R \mid S c=$ $0\}$ (resp., $\ell_{R}(S)=\{c \in R \mid c S=0\}$ ) which is called the right (resp., left) annihilator of $S$ in $R$.

Proposition 3.10. For an endomorphism $\sigma$ of a ring $R$, if $R$ is a $\sigma$-quasiArmendariz ring and $r_{R}(I)$ is a $\sigma$-ideal of $R$ for an ideal $I$ of $R$, then $R / r_{R}(I)$ is a $\bar{\sigma}$-quasi-Armendariz ring.

Proof. Let $\bar{a}=a+r_{R}(I)$ for $a \in R$. Suppose that $p(x)=\bar{a}_{0}+\bar{a}_{1} x+\cdots+\bar{a}_{m} x^{m}$, $q(x)=\bar{b}_{0}+\bar{b}_{1} x+\cdots+\bar{b}_{n} x^{n} \in\left(R / r_{R}(I)\right)[x ; \bar{\sigma}]$ with $p(x)\left(R / r_{R}(I)\right)[x ; \bar{\sigma}] q(x)=\overline{0}$. We claim that $\bar{a}_{i}\left(R / r_{R}(I)[x ; \bar{\sigma}]\right) \bar{b}_{j}=\overline{0}$ for each $i, j$. From

$$
p(x)\left(R / r_{R}(I)\right)[x ; \bar{\sigma}] q(x)=\overline{0},
$$

we get $p(x)\left(\bar{r} x^{t}\right) q(x)=\overline{0}$ for any $\bar{r} \in R / r_{R}(I)$ and nonnegative integer $t$. Hence for $0 \leq k \leq m+n, \sum_{i+j=k} a_{i} \sigma^{i}(r) \sigma^{t+i}\left(b_{j}\right) \in r_{R}(I)$, and so

$$
c \cdot \sum_{i+j=k} a_{i} \sigma^{i}(r) \sigma^{t+i}\left(b_{j}\right)=0
$$

for any $c \in I$. Thus $c a_{0} r \sigma^{t}\left(b_{0}\right) x^{t}+\left(c a_{0} r \sigma^{t}\left(b_{1}\right)+c a_{1} \sigma(r) \sigma^{t+1}\left(b_{0}\right)\right) x^{t+1}+\cdots+$ $c a_{m} \sigma^{m}(r) \sigma^{t+m}\left(b_{n}\right) x^{m+n+t}=\left(c a_{0}+c a_{1} x+\cdots+c a_{m} x^{m}\right)\left(r x^{t}\right)\left(b_{0}+b_{1} x+\cdots+\right.$ $\left.b_{n} x^{n}\right)=0$, and so $\left(c a_{0}+c a_{1} x+\cdots+c a_{m} x^{m}\right) R[x ; \sigma]\left(b_{0}+b_{1} x+\cdots+b_{n} x^{n}\right)=0$. Since $R$ is $\sigma$-quasi-Armendariz, we have $\left(c a_{i}\right) R[x ; \sigma] b_{j}=0$ for each $i, j$ and $c \in I$, and $a_{i} R[x ; \sigma] b_{j} \subseteq r_{R}(I)$. Hence $\bar{a}_{i}\left(R / r_{R}(I)[x ; \bar{\sigma}]\right) \bar{b}_{j}=\overline{0}$ for each $i, j$, and therefore $R / r_{R}(I)$ is $\bar{\sigma}$-quasi-Armendariz. 
Let $\sigma$ be an endomorphism of a ring $R$ and $e$ an idempotent of $R$ such that $\sigma(e)=e$. Then the map $\bar{\sigma}: e R e \rightarrow e R e$ defined by $\bar{\sigma}(e r e)=e \sigma(r) e$ for $r \in R$ is an endomorphism of $e R e$.

Proposition 3.11. Let $\sigma$ be an endomorphism of a ring $R$ and $e^{2}=e \in R$ with $\sigma(e)=e$. If $R$ is $\sigma$-quasi-Armendariz, then eRe is $\bar{\sigma}$-quasi-Armendariz.

Proof. Let $p(x)=\sum_{i=0}^{m} a_{i} x^{i}$ and $q(x)=\sum_{j=0}^{n} b_{j} x^{j}$ in $(e R e)[x ; \bar{\sigma}]$. Suppose that $p(x)(e R e)[x ; \bar{\sigma}] q(x)=0$. Note that $\bar{\sigma}(e)=e \sigma(e) e=e$, and so $p(x) e=p(x)$. For any $r \in R$ and nonnegative integer $t, p(x) r x^{t} q(x)=p(x)($ ere $) x^{t} q(x)=0$, and so $p(x) R[x ; \sigma] q(x)=0$. Since $R$ is $\sigma$-quasi-Armendariz, $a_{i} R[x ; \sigma] b_{j}=0$ for each $i, j$. Hence, for any nonnegative integer $t, 0=a_{i} R \sigma^{t}\left(b_{j}\right)=\left(a_{i} e\right) R \sigma^{t}\left(e b_{j}\right)$ $=a_{i}(e R e) \bar{\sigma}^{t}\left(b_{j}\right)$, since $p(x) e=p(x)$ and $e q(x)=q(x)$. Thus $a_{i}(e R e[x ; \bar{\sigma}]) b_{j}=$ 0 for each $i, j$, and therefore $e R e$ is $\bar{\sigma}$-quasi-Armendariz.

Corollary 3.12 ([5, Proposition 3.13]). If $R$ is a quasi-Armendariz ring, then for any nonzero idempotent $e \in R$, eRe is a quasi-Armendariz ring.

In [5, Theorem 3.16], it is proved that if $R$ is a quasi-Armendariz ring, then the polynomial ring $R[x]$ over $R$ is quasi-Armendariz. Finally, we extend this result and generalize the result of $[10$, Proposition 2.3] to a $\sigma$-quasi-Armendariz ring as follows.

Recall that if $\sigma$ is an endomorphism of a ring $R$, then the map $\bar{\sigma}: R[x] \rightarrow$ $R[x]$ defined by $\bar{\sigma}\left(\sum_{i=0}^{m} a_{i} x^{i}\right)=\sum_{i=0}^{m} \sigma\left(a_{i}\right) x^{i}$ is an endomorphism of the polynomial ring $R[x]$ and clearly this map extends $\sigma$.

Theorem 3.13. Let $\sigma$ be an automorphism of a ring $R$ with $\sigma^{t}=\mathrm{id}_{R}$ for some positive integer $t$. Then $R$ is a $\sigma$-quasi-Armendariz ring if and only if $R[x]$ is a $\bar{\sigma}$-quasi-Armendariz ring.

Proof. We extend the proof of [10, Proposition 2.3] to this case. Assume that $R$ is $\sigma$-quasi-Armendariz. Let $p(y)=f_{0}+f_{1} y+\cdots+f_{m} y^{m}$ and $q(y)=$ $g_{0}+g_{1} y+\cdots+g_{n} y^{n} \in(R[x])[y ; \bar{\sigma}]$ with $p(y)(R[x])[y ; \bar{\sigma}] q(y)=0$. We also let $f_{i}=a_{i_{0}}+a_{i_{1}} x+\cdots+a_{i_{w}} x^{i_{w}}, g_{j}=b_{j_{0}}+b_{j_{1}} x+\cdots+b_{j_{v}} x^{j_{v}} \in R[x]$ for each $0 \leq i \leq m$ and $0 \leq j \leq n$. We claim that $f_{i}(R[x][y ; \bar{\sigma}]) g_{j}=0$ for all $0 \leq i \leq m$ and $0 \leq j \leq n$. Take a positive integer $k$ such that $k=\sum_{i=0}^{m} \operatorname{deg}\left(f_{i}\right)+$ $\sum_{j=0}^{n} \operatorname{deg}\left(g_{j}\right)$, where the degree is considered as polynomials in $R[x]$ and the degree of zero polynomial is taken to be 0 . Let $p\left(x^{t k+1}\right)=f_{0}+f_{1} x^{t k+1}+\cdots+$ $f_{m} x^{m t k+m}$ and $q\left(x^{t k+1}\right)=g_{0}+g_{1} x^{t k+1}+\cdots+g_{n} x^{n t k+n} \in R[x ; \sigma]$. Then the set of coefficients of the $f_{i}^{\prime}$ s (resp., $g_{j}^{\prime}$ s) equals the set of coefficients of $p\left(x^{t k+1}\right)$ (resp., $\left.q\left(x^{t k+1}\right)\right)$. Since $p(y)(R[x])[y ; \bar{\sigma}] q(y)=0$, we have also $p(y) r y^{s} q(y)=0$ for any $r \in R$ and nonnegative integer $s$. Then $f_{0} r \bar{\sigma}^{s}\left(g_{0}\right) y^{s}+\left(f_{0} r \bar{\sigma}^{s}\left(g_{1}\right)+\right.$ $\left.f_{1} \bar{\sigma}(r) \bar{\sigma}^{s+1}\left(g_{0}\right)\right) y^{s+1}+\cdots+f_{m} \bar{\sigma}^{m}(r) \bar{\sigma}^{s+m}\left(g_{n}\right) y^{s+m+n}=0$. This implies that $\left(f_{0}+f_{1} x^{t k+1}+\cdots+f_{m} x^{m t k+m}\right) r x^{s}\left(g_{0}+g_{1} x^{t k+1}+\cdots+g_{n} x^{n t k+n}\right)=0$. Hence $p\left(x^{t k+1}\right) R[x ; \sigma] q\left(x^{t k+1}\right)=0$. Since $R$ is $\sigma$-quasi-Armendariz, $a_{\alpha} R[x ; \sigma] b_{\beta}=0$ for each $0 \leq i \leq m, 0 \leq j \leq n, 0 \leq \alpha \leq i_{w}$ and $0 \leq \beta \leq j_{v}$. Thus 
$f_{i}(R[x][y ; \bar{\sigma}]) g_{j}=0$ for all $0 \leq i \leq m$ and $0 \leq j \leq n$. Therefore $R[x]$ is $\bar{\sigma}$-quasi-Armendariz.

Conversely, assume that $R[x]$ is $\bar{\sigma}$-quasi-Armendariz. Let $p(x)=\sum_{i=0}^{m} a_{i} x^{i}$ and $q(x)=\sum_{j=0}^{n} b_{j} x^{j}$ in $R[x ; \sigma]$ such that $p(x) R[x ; \sigma] q(x)=0$. Since

$$
p(x) r x^{s} q(x)=0
$$

for any $r \in R$ and nonnegative integer $s$, we have $a_{0} r \sigma^{s}\left(b_{0}\right)=0, a_{0} r \sigma^{s}\left(b_{1}\right)+$ $a_{1} \sigma(r) \sigma^{s+1}\left(b_{0}\right)=0, \ldots, a_{m} \sigma^{m}(r) \sigma^{s+m}\left(b_{n}\right)=0$. Let $p(y)=a_{0}+a_{1} y+\cdots+$ $a_{m} y^{m}, q(y)=b_{0}+b_{1} y+\cdots+b_{n} y^{n} \in(R[x])[y ; \bar{\sigma}]$. For any $r \in R$ and nonnegative integer $s, p(y) r y^{s} q(y)=a_{0} r \bar{\sigma}^{s}\left(b_{0}\right) y^{s}+\left(a_{0} r \bar{\sigma}^{s}\left(b_{1}\right)+a_{1} \bar{\sigma}(r) \bar{\sigma}^{s+1}\left(b_{0}\right)\right) y^{s+1}+\cdots+$ $a_{m} \bar{\sigma}^{m}(r) \bar{\sigma}^{s+m}\left(b_{n}\right) y^{m+n+s}=0$. Thus $p(y) R[y ; \bar{\sigma}] q(y)=0$, and so

$$
p(y)(R[x])[y ; \bar{\sigma}] q(y)=0
$$

because $y x=x y$. Since $R[x]$ is $\bar{\sigma}$-quasi-Armendariz, we have $a_{i}(R[x][y ; \bar{\sigma}]) b_{j}=$ 0 for all $i, j$ and so $a_{i} R[x ; \sigma] b_{j}=0$. Thus $R$ is $\sigma$-quasi-Armendariz.

Acknowledgments. The authors thank the referee for his/her very careful reading of the manuscript. The second named author was supported by Basic Science Research Program through the National Research Foundation of Korea(NRF) funded by the Ministry of Education, Science and Technology(No. 2010-0022160).

\section{References}

[1] E. P. Armendariz, A note on extensions of Baer and P.P.-rings, J. Austral. Math. Soc. 18 (1974), 470-473.

[2] M. Başer, A. Harmanci, and T. K. Kwak, Generalized semicommutative rings and their extensions, Bull. Korean Math. Soc. 45 (2008), no. 2, 285-297.

[3] M. Başer, F. Kaynarca, T. K. Kwak, and Y. Lee, Weak quasi-Armendariz rings, to apperar in Algebra Colloq.

[4] W. Cortes, Skew Armendariz rings and annihilator ideals of skew polynomial rings, Algebraic structures and their representations, 249-259, Contemp. Math., 376, Amer. Math. Soc., Providence, RI, 2005.

[5] Y. Hirano, On annihilator ideals of a polynomial ring over a noncommutative ring, J. Pure Appl. Algebra 168 (2002), no. 1, 45-52.

[6] C. Y. Hong, N. K. Kim, and T. K. Kwak, Ore extensions of Baer and p.p.-rings, J. Pure Appl. Algebra 151 (2000), no. 3, 215-226.

[7] _ On skew Armendariz rings, Comm. Algebra 31 (2003), no. 1, 103-122.

[8] _ On quasi-rigid ideals and rings, Bull. Korean Math. Soc. 47 (2010), no. 2, 385-399.

[9] C. Y. Hong, N. K. Kim, and Y. Lee, Skew polynomial rings over semiprime rings, J. Korean Math. Soc. 47 (2010), no. 5, 879-897.

[10] C. Y. Hong, T. K. Kwak, and S. T. Rizvi, Extensions of generalized Armendariz rings, Algebra Colloq. 13 (2006), no. 2, 253-266.

[11] A. A. M. Kamal, Some remarks on Ore extension rings, Comm. Algebra 22 (1994), no. 10, 3637-3667.

[12] N. K. Kim and Y. Lee, Armendariz rings and reduced rings, J. Algebra 223 (2000), no. $2,477-488$.

[13] J. Krempa, Some examples of reduced rings, Algebra Colloq. 3 (1996), no. 4, 289-300.

[14] T. Y. Lam, A First Course in Noncommutative Rings, Springer-Verlag, New York, 1991. 
[15] T. K. Lee and Y. Q. Zhou, Armendariz and reduced rings, Comm. Algebra 32 (2004), no. $6,2287-2299$.

[16] J. Matczuk, A characterization of $\sigma$-rigid rings, Comm. Algebra 32 (2004), no. 11, 4333-4336.

[17] K. R. Pearson and W. Stephenson, A skew polynomial ring over a Jacobson ring need not be a Jacobson ring, Comm. Algebra 5 (1977), no. 8, 783-794.

[18] M. B. Rege and S. Chhawchharia, Armendariz rings, Proc. Japan Acad. Ser. A Math. Sci. 73 (1997), no. 1, 14-17.

Muhittin BAŞER

Department of Mathematics

KocAtepe University

Afyonkarahisar 03200, Turkey

E-mail address: mbaser@aku.edu.tr

Tai Keun KWaK

Department of Mathematics

DAEJin University

PoCHEON 487-711, KorEA

E-mail address: tkkwak@daejin.ac.kr 